\title{
Aspekte der österreichischen staatlichen Verwaltung in Dalmatien am Beginn des 20. Jh.
}

\begin{abstract}
Aspects of the Austrian State Administration in Dalmatia at the Beginning of the $20^{\text {th }}$ Century
Looking at the old Austrian political and state administration, you can largely describe it as elegant. Although its administrative organs in Dalmatia and Vienna also had to safeguard general state interests, they were largely objective. They operated basically supranationally, knew how to deal with crises in a country, tried to balance interests between disputing parties, performed mediation tasks and rejected rather inappropriate desires on the part of the military administration. The success of a smooth administration which saw itself as a hinge between the interests of Vienna and Dalmatia depended to a large extent on the spirit of the various Statthalter (civil representatives of the crown land). These Austrian administrative efforts can only be recognized through an intensive study of unprinted government administrative files.
\end{abstract}

Keywords: administrative history - civil representatives of the crown land - Dalmatia - Habsburg monarchy

\section{Einleitung}

Dalmatien, das 634.855 Personen einheimischer Bevölkerung im Jahr 1910 zählte, war neben Salzburg das jüngste Kronland. Beide Kronländer wurden, nach einem kurzen Intermezzo im Zuge der napoleonischen Kriege, am Wiener Kongress der österreichischen Reichshälfte der Habsburgermonarchie definitiv angeschlossen. ${ }^{1}$ Dalmatien war mit einem Steueraufkommen von 18 Kronen (K.) pro Kopf und Jahr (1904) auch das ärmste Kronland; danach folgten Galizien $(21 \mathrm{~K}$.) und die Bukowina ( $25 \mathrm{~K}$.). Im Vergleich dazu hatten die beiden Kronländer mit der höchsten Steuerleistung ein Aufkommen pro Person von 108 K. (Niederösterreich) bzw. 62 K. (Salzburg). ${ }^{2}$ Diese Armut hatte u.a. mit der agrarischen Prägung und der geopolitischen Position des Landes $\mathrm{zu}$ tun. In gewissen verwaltungs-

\footnotetext{
${ }^{1}$ STERGAR, Nationswerdungsprozesse 101.

${ }^{2}$ G. FREYTAG's Reichsrats-Wahlkarte.
}

technischen Belangen stand Dalmatien hingegen nicht unbedingt an letzter Stelle, mitunter war es besser gestellt als andere der 13 staatlichen Landesverwaltungseinheiten. So lag es z.B. hinsichtlich des Anteils an Postämtern mit Telegraphenanschluss mit 75 Prozent im Mittelfeld (Salzburg: $94 \%$, Galizien: $50 \%$ ), auch hinsichtlich der Versorgung mit Postämtern. Bezüglich der Dichte der untersten staatlichen Verwaltungsstruktur nahm Dalmatien mit 14 Bezirkshauptmannschaften $^{3}$ den vierten Rang von oben ein (durchschnittlich 45.347 Einwohner pro Bezirkshauptmannschaft), hinter Salzburg (ca. 34.800), Tirol (35.000) und Krain (43.400). Wohl verkehrstechnisch zu begründen ist der Umstand, dass Dalmatien ein eigenes Oberlandesgericht besaß (so auch das Küstenland, ca. 830.000 Einwohner), während das OLG in Graz die Länder Steiermark, Kärnten und Krain (ca. 2,3 Mio. Einwohner) oder das OLG in Wien die Länder Nieder-

\footnotetext{
${ }^{3}$ Siehe Text bei Fn. 27.
} 
österreich, Oberösterreich und Salzburg zu betreuen hatten (ca. 4,3 Mio. Einwohner). Auf kirchlicher Verwaltungsebene gab es in Dalmatien das engmaschigste Netz an katholischen Diözesen. Die Katholiken (83,5\%) hatten ein Erzbistum in Zara [Zadar] sowie fünf weitere Bistümer (in Cattaro [Kotor], Lesina [Hvar], Ragusa [Dubrovnik], Sebenico [Šibenik], Spalato [Split]-Makarska), somit ca. 106.000 Katholiken pro Diözese. Dazu kamen zwei griechisch-orientalische Bistümer (eines in Zara für Dalmatien und Istrien, das andere für Cattaro und Ragusa). Nur halb so privilegiert waren die zweitgereihten Katholiken des Küstenlands (ca. 203.000 Katholiken pro Diözese), am geringsten war die Dichte in Niederösterreich bzw. Böhmen (ca. 1,5 bzw. 1,6 Mio. Katholiken pro Diözese). ${ }^{4}$

Der vorliegende Artikel präsentiert Erkenntnisse der noch nicht publizierten Dissertation des Autors. ${ }^{5}$ In ihr wird das Beziehungsgeflecht zwischen staatlich-politischer Verwaltung und politischer Repräsentanz Dalmatiens im frühen 20. Jahrhundert betrachtet. Gleichzeitig will sie eine Lücke in der deutschsprachigen wissenschaftlichen Literatur zwischen den Forschungsergebnissen von Günter Schödl (bis 1907) und Aleksandar Jakir (ab 1918) zu Politik und Verwaltung verkleinern. ${ }^{6}$ Dies erfolgt einerseits im Überblick zur politischen Geschichte Dalmatiens, andererseits mittels sog. „exemplarischer Tiefenbohrung" bei einzelnen Themen, um das Agieren der Beteiligten näher unter die Lupe zu nehmen (z.B. anhand der Reichsrats- und Land-

\footnotetext{
${ }^{4}$ Die Berechnungen erfolgten aufgrund der Angaben im Hof- und Staats-Handbuch 1015ff., 451ff., 576ff., 594ff., 604ff., 642ff., 652ff., 663ff., 694ff., 729ff., 849ff., 895ff., 907ff., 998ff. Sie sind daher nicht exakt, geben aber Annäherungswerte an. Bei den Berechnungen zu den Bezirkshauptmannschaften sind Statutarstädte den Bezirkshauptmannschaften gleichgestellt.

${ }^{5}$ KalWODA, Parteien. Die Publikation ist für 2021 geplant.

${ }^{6}$ SCHÖDL, Kroatische Nationalpolitik; JAKIR, Dalmatien zwischen den Weltkriegen.
}

tagswahlen, des Sprachenausgleichs oder der Gemeinderatsauflösungen). ${ }^{7}$

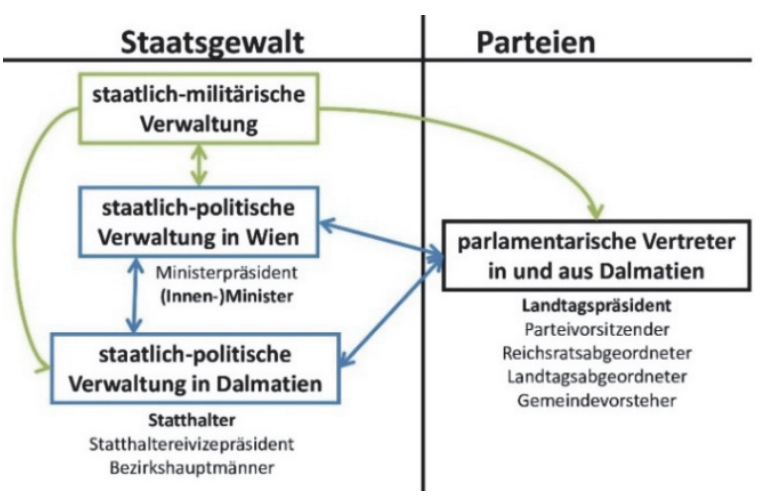

Abb. 1: Beziehungsgeflecht zwischen

Staatsgewalt und Parteien

In diesem Aufsatz sei zunächst auf den Kern des dalmatinischen Parteienwesens zwischen 1900 und 1918 eingegangen, bevor wesentliche Elemente staatlichen Agierens derselben Zeitspanne besprochen werden. Eine sog. exemplarische Tiefenbohrung bildet den Abschluss dieser Arbeit. Gerade Letztere belegt, dass die staatlichösterreichische Verwaltung Dalmatiens vielfach negativ gesehen wird. Dies ist deshalb der Fall, weil von dalmatinischen Politikern vorwiegend Defizite aufgezeigt und diese vielfach überzeichnet worden sind. Diese politische Sicht auf die österreichische Verwaltung wird von der Historiographie dann übernommen, wenn sie vorrangig auf Egodokumente von Politikern oder auf politische Äußerungen zurückgreift, die in Zeitungen oder Stenographischen Protokollen wiedergegeben sind (vgl. Kap.4). Eine neue Perspektive auf die österreichische Verwaltung ist dann zu gewinnen, wenn, ganz im Sinne von Ivan Pederin, die dalmatinische Geschichte auch ",aus der Sicht des Statthalters und der Wiener Ministerien" betrachtet wird. ${ }^{8}$

\footnotetext{
${ }^{7}$ KALWODA, Parteien.

${ }^{8}$ Pederin, Austrijska vlast $7 \mathrm{f}$.
} 


\section{Parteien(-interessen)}

Das dalmatinische Parteiengefüge ab 1900 ist nicht linear darstellbar. Die Zeitspanne bis zum Untergang der Monarchie war von unterschiedlichen Bündnissen zwischen Parteien, von Abspaltungen und Fusionen gekennzeichnet. Details sind hier nicht aufrollbar. ${ }^{9}$ Von Interesse ist die Frage, welche grundsätzlichen Forderungen parlamentarische Vertreter gegenüber der staatlichen Verwaltung aufstellten. Hier gab es, je nach Partei- und Nationszugehörigkeit, unterschiedliche Ziele.

Ziel aller Dalmatiner, unabhängig von der Nationalitätszugehörigkeit, war der Wunsch, dass die Staatsverwaltung massiv in die Infrastruktur und in die wirtschaftliche Hebung des Landes investiere. Da gab es eine lange Latte an konkreten Verbesserungsvorschlägen. Die Eisenbahnverbindungen innerhalb des Landes und über seine Grenzen hinaus, der Straßenausbau, die Erhaltung von Kulturgütern und Kirchen, die Intensivierung des Fremdenverkehrs oder die Förderung der Landwirtschaft seien hier exemplarisch erwähnt.

Weiters gab es Ziele aller Kroaten, die zunächst nicht unbedingt auch von den Serben geteilt, von Italienern aber entschieden abgelehnt wurden. Dazu zählten v.a. national-politische Forderungen. An oberster Stelle der Wunschliste stand die Vereinigung der kroatischen Länder von Zis- und Transleithanien, dem sich in der Resolution von Zara ${ }^{10} 1905$ auch die Serben anschlossen. Spätestens im Verlauf des Ersten Weltkriegs etablierte sich ein slawisch-parteiübergreifender Konsens nach Einigung aller südslawischen Gebiete der Monarchie im trialistischen Sinne. Diese Forderungen scheiterten am

\footnotetext{
${ }^{9}$ ScHÖDL, Kroatische Nationalpolitik; KALWODA, Politik; JAKIR, TROGRLIĆ, Dalmatien.

${ }^{10}$ AVA MdI-Präs 22/gen, Kart. 2012, 7009-1905, Nardelli an MdI (20. 10. 1905). Zum Wortlaut der Resolution z.B. BAKOTIĆ, Srbi u Dalmaciji 142 f.
}

österreichisch-ungarischen Dualismus. Eine weitere national-politische Forderung der dalmatinischen Slawen war, ein Ende der Bevorzugung alles Italienischen im Land zu erzielen, v.a. die innere italienische Dienstsprache bei den staatlichen Behörden zu beseitigen.

Freilich verfolgten die slawisch-dalmatinischen Parteien auch unterschiedliche Ziele. Die Landesrepräsentanz (Landtag, Dalmatinski sabor) blieb, anders als in anderen Kronländern der Monarchie, eine Interessensvertretung ohne eine allgemeine Wählerkurie. ${ }^{11}$ Letztere wurde, trotz Verhandlungen durch viele Jahre hindurch, nicht eingeführt, weil sie Vertreter der Italienischen Partei [Partito autonomo italiano] und der Kroatischen Partei [Hrvatska stranka] verhinderten. Die Kroatische Partei galt lange Zeit als konservative Honoratiorenpartei, die mit großen Mandatseinbußen zugunsten der Reinen Rechtspartei [Čista stranka prava] bzw. der JosipSmodlaka-Partei (Kroatische Volks- und Fortschrittspartei [Hrvatska pučka napredna stranka]) rechnen musste. Sozial bedingte Interessensgegensätze zeigten sich auch in unterschiedlichen wirtschaftspolitischen Forderungen kroatischer Parteien. An der Beseitigung bzw. Milderung der Auswirkungen des Kolonatwesens ${ }^{12}$ hatten die besitzenden Gesellschaftsschichten, die vornehmlich in der Kroatischen und in der Italienischen Partei vertreten waren, wenig Interesse. So gesehen verwundert die Aussage des aus Böhmen stammenden, tschechisch sozialisierten dalmatinischen Statthaltereivizepräsidenten Franz von Thun-Hohenstein vom Febru-

\footnotetext{
${ }^{11}$ Es bestanden die Wählerkurien der Höchstbesteuerten, Handelskammern, Städte, und Landgemeinden. PERIĆ, Dalmatinski sabor; BUCZYNSKI, Der dalmatinische Landtag.

${ }^{12}$ Die Kolonen (Landwirtschaft betreibende, persönlich freie, wirtschaftlich vielfach von einem Grundherrn abhängige Pächter) waren bestrebt, die Abgabenquoten an den Grundeigentümer zu reduzieren. SCHULLERN-SCHRATTENHOFEN, Kolonat; SCHWIEDLAND, Interministerielle Dalmatinische Kommission.
} 
ar 1912 nicht, dass der Landtag in Dalmatien „hier ebenso wenig vom Fleck wie der in Böhmen u[nd] vielen anderen Ländern" gehe. ${ }^{13}$

Die dalmatinischen Parteien unterschieden sich ganz wesentlich in der Form, wie sie die politischen Ziele erreichen wollten. Die einen versuchten es über die sog. positive Politik, um sich im Konsens mit der Staatsverwaltung den politischen Zielen in behutsamen Schritten zu nähern, ohne Wien zu verärgern (beispielsweise in der Frage der Dualismusüberwindung). Die Regierung in Wien wiederum, oft bloß auf eine knappe Mehrheit im Abgeordnetenhaus des Reichsrats gestützt, war gerade ab der Obstruktionsphase sehr auf diese Positivisten angewiesen, wollte sie Gesetzesvorlagen durchbringen. Die andere Gruppe an Abgeordneten übte sich in der Fundamentalopposition gegenüber Wien bzw. der staatlichen Verwaltung im Land und wurde hiebei teilweise von politischen Versprechungen aus Ungarn gelockt.

\section{Das staatliche Agieren}

Die Akteure auf Seiten der staatlichen Verwaltung sind in hierarchischer Gliederung leicht dingfest zu machen: In der Zentralregierung in Wien spielten Ministerpräsidenten und von den Ministern v.a. die Innenminister eine wesentliche Rolle. Auf Landesebene waren es allen voran die jeweiligen Statthalter (Emil von DavidRhonfeld, Erasmus von Handel, Nikolaus Nardelli, Marius von Attems-Heiligenkreuz) mit ihrem Stab in der Statthalterei in Zara, wozu an vorderster Front der Statthaltereivizepräsident (Stellvertreter) und der Präsidialist zählten. Eine weitere Ebene darunter waren als verlängerter Arm bzw. als Zuarbeiter der Statthalterei die

\footnotetext{
${ }^{13}$ Státní oblastní archiv Litoměřice, Zweigstelle Děčín, FA Thun-Hohenstein, Linie Tetschen: NL Franz Thun, A-3 XXIII, H I/a, Nr. 41, Franta an Franz Thun (3. 2. 1912).
}

Bezirkshauptmänner angesiedelt. Im staatlichen Agieren lassen sich nun mehrere Funktionen wahrnehmen, von denen folgende herausgenommen werden.

Sowohl die Ministerien in Wien als auch die Statthalterei arbeiteten supranational, indem sie auf berechtigte Forderungen von Nationalitäten eingingen, Forderungen aber ablehnten, wenn sie aus gesamtstaatlicher Sicht nicht rechtfertigbar waren oder eine Bevorzugung einer Nationalität bedeutet hätten. So verlangten z.B. 1906 die dalmatinischen Italiener (knapp 3 Prozent der Bevölkerung) einen eigenen Reichsratsabgeordneten bei den Verhandlungen um die neue Reichsratswahlordnung oder 1909 größere Zugeständnisse bei den Ausnahmsbestimmungen hinsichtlich der Sprachenverordnung, bevor in der Verordnung vom April 1909 in Dalmatien das Kroatische bzw. Serbische als innere Dienstsprache bei den staatlichen Behörden anstelle des Italienischen eingeführt wurde. Die Regierung lehnte die italienischen Ansinnen ab, weil dies die numerische Größe der Italiener in Dalmatien nicht rechtfertige. Die gestatteten Ausnahmsbestimmungen sollten dem „spezifischen Gewicht" (der sozialen und kulturellen Bedeutung) der Italiener Rechnung tragen..$^{14}$ Andererseits schützten Statthalter und Minister im Ersten Weltkrieg die Minderheitenrechte der Italiener auch nach dem Kriegseintritt von Italien. Hier gab es Wünsche der Kroaten oder der Militärverwaltung, italienische Ortsbezeichnungen durch kroatische zu ersetzen, alle italienischen Vereine aufzulösen oder die Ausnahmsbestimmungen zugunsten der Italiener in der Sprachenverordnung von 1909 zu streichen. ${ }^{15}$

Die Statthalterei sah sich als Bindeglied zwischen Wiener Zentrale und gewählten Repräsentanten Dalmatiens, weshalb sie sich um einen

\footnotetext{
${ }^{14}$ StPrAH-Beil., 17. Sess., Nr. 2727 (12. 6. 1906), 240f.; KALWODA, Parteien 94-96, 182, 187, 208; FremdenBlatt Nr. 154 v. 6. 6. 1909, Morgen-Blatt 1.

${ }^{15}$ KALWODA, Parteien 286f.
} 
Ausgleich zwischen beiden Interessenspolen bemühte. Dies hieß, der Statthalter intervenierte wiederholt und vehement in Wien, dass einerseits dalmatinische Landesinteressen umgesetzt werden. Er tat dies andererseits aber auch, um mögliche Nachteile von der staatlichen Verwaltung fernzuhalten, damit z.B. radikalen Abgeordneten nicht politische Munition geliefert wurde, die sich gegen die Regierung oder gegen weniger oppositionelle Abgeordnete richtete. Wien reagierte meistens im Sinne des Landes, wenn auch mitunter zeitverzögert. Die Belege für eine Intervention der Statthalter zugunsten des Landes sind zahlreich. Beispielsweise forderten sie bei den dalmatinischen Parteien und in Wien stets aufs Neue ein Weiterverhandeln ein, damit eine neue Regelung des Sprachengebrauchs bei den staatlichen Zivilbehörden in Dalmatien vorangetrieben werde. ${ }^{16} \mathrm{Im}$ Jänner 1909 richtete Nardelli wiederum an Wien den Appell, die Verhandlungen zum Sprachengebrauch auch zum Abschluss zu bringen, damit die Regierung bei einer weiteren Verzögerung nicht Gefahr laufe, „die Sympathien der Kroaten und Serben im Lande, welche bereits durch 30 Jahre auf die Realisierung dieser ihrer Forderung warten, gänzlich" zu verwirken. ${ }^{17}$ Allen staatlichen Verwaltungsebenen war aber auch klar, dass die Sprachenfrage vor Einberufung des Landtags (diese erfolgte im Herbst 1909) zu lösen sei, weil sonst „ein normales Funktionieren des dalmatinischen Landtages kaum zu erwarten" gewesen wäre. ${ }^{18}$

\footnotetext{
${ }^{16}$ Ebd. 176-186; z.B. auch AVA MdI-Präs 3/Sprache, Kart. 1307, 6419-1908, Nardelli an MdI (9. 7. 1908).

${ }^{17}$ AVA MdI-Präs 3/Sprache, Kart. 1308, 741-1909, Nardelli an MdI (12. 1. 1909) sub 742-1909; ähnlich unter ebd., 742-1909, Nardelli an MdI (14. 1. 1909); ebd., 2670-1909, Nardelli an MdI (26. 2. 1909); AVA MRP, Kart. 108, 4866-1908 (2. 11. 1908) sub 4124-1910. ${ }^{18}$ AVA MdI-Präs 3/Sprache, Kart. 1308, 2888-1909, Nardelli an MdI (2. 3. 1909); Neue Freie Presse, Nr. 13990 v. 25. 2. 1909, Abendblatt 3; AVA MRP, Kart. 108, 4866-1908 (2. 11. 1908) sub 4124-1910.
}

Die Statthalterei wirkte weiters auf Abgeordnete ein, damit der Gesamtstaat leichter seine Interessen wahren konnte. So nahmen sie z.B. im Vorfeld von Wahlen Einfluss zugunsten weniger oppositioneller oder konservativer Reichsratskandidaten, damit die Regierung von Abgeordneten ferngehalten werde, die ihr durch Wortmeldungen oder Stimmverhalten im Parlament Verlegenheit bereiten könnten. Doch taten dies nicht alle Statthalter, so Statthalter Handel nicht, als ihn Teile der politischen Elite vor der Reichsratsergänzungswahl von 1904 baten, den oppositionellen Kandidaten Ante Tresić-Pavičić zu verhindern. Handel griff in Wahlvorbereitungen aus prinzipiellen Gründen nicht ein. ${ }^{19}$ Beim Thema Sprachengebrauch bei den staatlichen Zivilbehörden beharrten die Statthalter wiederum auf der Einhaltung der innersten deutschen Dienstsprache im Verkehr mit Zentralstellen in Wien. Während dies Statthalter Handel in seinem Regierungsentwurf explizit tat und ihm deshalb ein Entrüstungssturm von allen dalmatinischen Parteien entgegenblies, bestand Nardelli lediglich implizit darauf und bekam dafür auch den Segen der maßgeblichen dalmatinischen Verhandler. ${ }^{20}$

Die Statthalter und die Regierung in Wien nahmen zusehends eine Mediatorenfunktion zwischen den Parteien ein, wenn Parteien zwar Veränderungen in ihrem Verhältnis zueinander anstrebten, sich über das Wie aber nicht einigen konnten. So gingen Abänderungswünsche von Landtagswahlordnungen von den jeweiligen Landtagen selbst aus. Auch die dalmatinischen Parteien (die Initiative lag v.a. bei der Reinen Rechtspartei und der Smodlaka-Partei) bemühten sich um einen Kompromiss der unterschiedlichen Interessen, beispielsweise in den Belangen der Wahlrechtserweiterung oder der Besei-

\footnotetext{
${ }^{19}$ KALWODA, Staatliche Einflussnahme; Ders., Parteien $49 f$.

${ }^{20}$ Trogrlić, Erazmo Handel 93-96; KalwOdA, Parteien 176f., 178-192.
} 
tigung des Kolonatwesens. Kurz vor Ausbruch des Weltkriegs stand man vor einer Einigung, schaffte diese aber nicht bis zum Schlusspunkt. Als Statthalter Attems Parteivertretern anbot, die Statthalterei werde einen Kompromissvorschlag als Entwurf ausarbeiten, wurde dies von allen Parteien begrüßt. Ende April 1914 war er fertig. Der Krieg machte ein Zuendeverhandeln unmöglich. ${ }^{21}$

Hinsichtlich des Verhältnisses zwischen staatlich-politischer und staatlich-militärischer Verwaltung ist vor dem Weltkrieg zu beobachten, dass die politische Verwaltung allzu große zentralistische Begehrlichkeiten der militärischen Verwaltung nicht aufkommen ließ. Als im Vorfeld des Sprachenausgleichs von 1909 in einer interministeriellen Konferenz das Reichskriegsund das Landesverteidigungsministerium von der staatlich-politischen Verwaltung in Dalmatien einforderten, alle administrativen Ausfertigungen von militärischem Interesse hätten samt betreffenden Akten und Beilagen ins Deutsche übersetzt zu werden, verwies der Innenminister auf die politische und administrative Undurchführbarkeit des Ansinnens. Er sagte lediglich zu, sich dafür einzusetzen, dass der wesentliche Inhalt eines Gegenstandes in deutscher Übersetzung Militärbehörden zugeschickt werde. Die Zusammenarbeit dieser beiden staatlichen Pole war während des Weltkriegs allerdings ambivalent: Die Statthalterei arbeitete am Beginn des Ersten Weltkriegs eng und kritiklos mit der Militärverwaltung zusammen. Dies änderte sich aber relativ rasch und Statthalter Attems bekam dabei stets Rückendeckung aus Wien.22

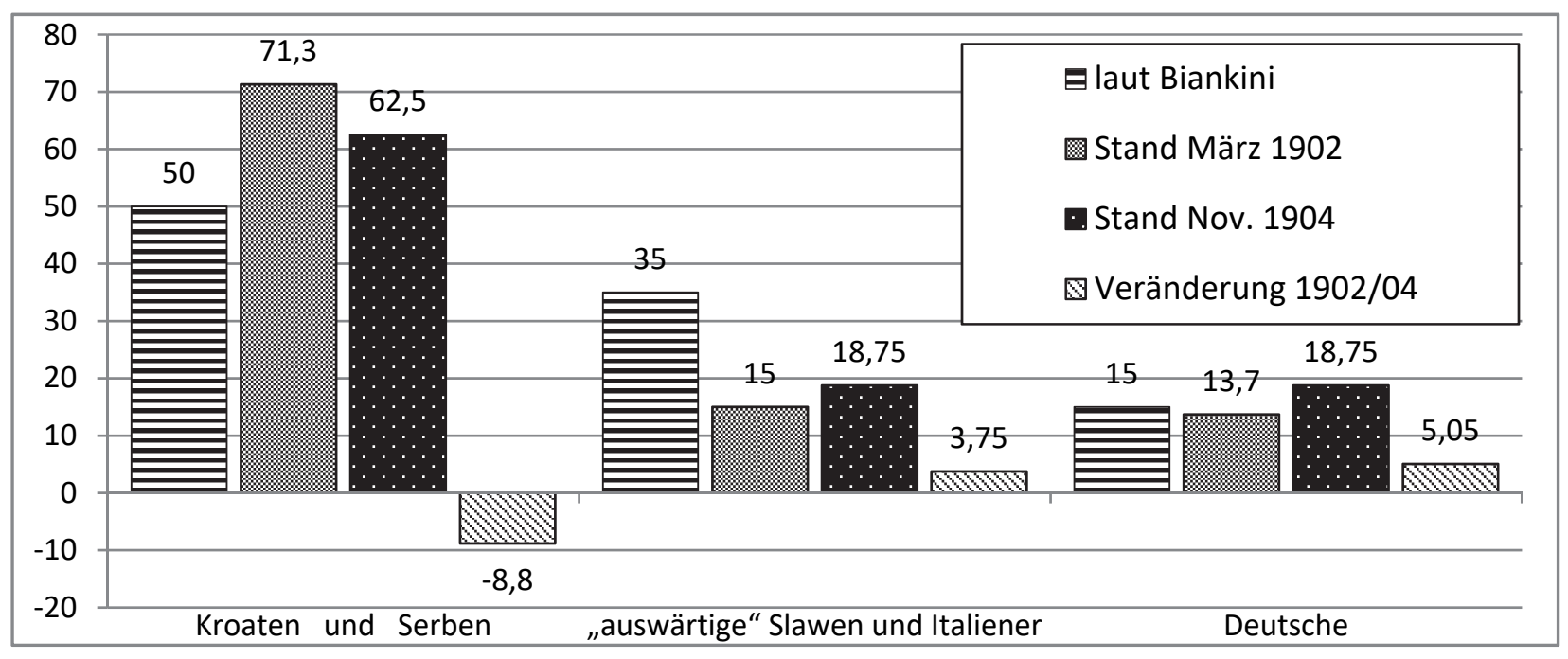

Abb. 2: Relative Verteilung der staatlich-politischen (Konzepts-)Beamten in Dalmatien

\footnotetext{
${ }^{21}$ KALWODA, Parteien 264-267; AVA MdI-Präs 31/Dalm, Kart. 2175, MF 33, 6996-1914 sub 7015-1914, Statth. Telegramm an MdI (21. 6. 1914); ebd., 70151914, Attems an MdI (21. 6. 1914).
}

22 AVA MdI-Präs 3/Sprache, Kart. 1308, 248-1909, Protokoll interministerielle Beratung (7. 1. 1909); KALWODA, Engherzige Beurteilung; KALWODA, Nationale Schlagseiten; KALWODA, Parteien 270-276, 278281, 319f. 


\section{Germanisierende Statthalterei?}

Was den Ruf der Statthalterei und der Staatsgewalt im Land angeht, so war dieser viel schlechter, als sie es verdient hätten. Dafür gab es zwei Gründe: Vielfach sah man dalmatinischerseits die Bemühungen der Statthalterei um die Wohlfahrt des Landes nicht, weil man dies teils auch nicht wollte. Auf der anderen Seite bemühten sich auch staatliche Stellen zu wenig, ihr Wirken für das Land gehörig publik $\mathrm{zu}$ machen, wodurch das "Marketing“ in eigener Sache zu wenig nachhaltig war. Beides lässt sich anhand des nachfolgenden Beispiels belegen:

Die Statthalterei war wiederkehrend mit dem Vorwurf konfrontiert, sie betreibe eine Personalpolitik, die die staatlich-dalmatinische Verwaltung germanisiere. Abgeordneter Juraj Biankini unterlegte 1904 diese These auch mit Zahlen. Er behauptete im Abgeordnetenhaus, in der politischen Verwaltung Dalmatiens gebe es „50 Prozent fremde Beamte“ und „von diesen mindestens 30 Prozent Deutsche", "von welchen aber 20 Prozent die kroatische Sprache nicht kennen. ${ }^{23}$ Diese Zahlen Biankinis sind zwar falsch, die Relationen, die er aufzeigt, aber nicht ganz. Faktum war, der Anteil der kroatischen und serbischen Dalmatiner bei den politischen Konzeptsbeamten sank in einem Zeitraum von fast drei Jahren (zwischen März 1902 und Ende November 1904) von 71,3 auf 62,5 Prozent. ${ }^{24}$ Der Anteil der deutschen Konzeptsbeamten wiederum stieg von fast 14 auf 19 Prozent. In diesem Zeitraum vermehrte sich das Konzeptsbeamtenpersonal in Summe zwar von 80 auf 96, aber bei Kroaten und Serben nicht in dem Ausmaß wie bei den Kategorien "auswärtige Slaven und Italiener" bzw. Deutsche. Somit kann festgehal-

${ }^{23}$ Biankini: StPrAH, 17. Sess. (23. 11. 1904), 25668.

${ }^{24}$ Basis der Berechnung von Herkunft und Nationalität der Beamten seitens der Statthalterei waren die Personalstandesausweise: AVA MdI-Präs 4/Dalm, Kart. 1395, MF 4, 8442-1904, Handel an MdI (27. 11. 1904). ten werden, Biankinis Relationen stimmen in der Grundaussage, dass der Anteil der Slawen abgenommen habe. Ganz falsch war aber Biankinis Sicht auf das Motiv, das er der österreichischen Verwaltung unterstellte, wenn er festhielt, die Statthalterei germanisiere absichtlich die staatliche Verwaltung in Dalmatien. Diese Sicht Biankinis übernahm mitunter die jüngere Literatur sehr unkritisch, wenn sie sich ausschließlich auf gedruckte Quellen bezog. ${ }^{25}$

Der Grund für die prozentuelle Abnahme an slawischen Beamten war das geringe Interesse von Slawen am politischen Staatsdienst, auch an der Justizverwaltung, das es unmöglich machte, „alle systemisierten Posten des politischen Konzeptsdienstes“ zu besetzen. Erkrankungen, Todesfälle, Erschöpfungszustände aufgrund von Arbeitsüberlastung und Ausfälle aufgrund von Krankheiten wie Malaria- oder Fieberbefall verschärften darüber hinaus die misslichen Personalverhältnisse. Die Statthalterei sah in der Überbürdung der politischen Beamten einen Mitgrund, „dass sich von heimischen sprachenkundigen jungen Leuten Niemand diesem Dienste“ zuwandte. ${ }^{26}$

Diesem Trend trachtete die Statthalterei vor und zur Jahrhundertwende gegenzusteuern. Sie schnürte ein Bündel an Maßnahmen zur Behebung des Mangels an Nachwuchs Einheimischer. Dabei wurde sie in ihren Bemühungen vom Innenministerium tatkräftig unterstützt.

Zum einen sollten günstigere Beförderungsaussichten herbeigeführt werden. Die Statthalterei begründete dies damit, dass sich die politischen Beamten aufgrund der "nicht behebbaren Schwierigkeiten und Widrigkeiten" des Dienstes dadurch "wenigstens zum Theile schadlos" halten könnten. Dazu gehörte jedenfalls, diverse Posten neu zu systemisieren. Statthalter David

\footnotetext{
${ }^{25}$ Unkritisch z.B. bei LACMANOVIĆ-HEYDENREUTER, Dalmatien 198-203.

${ }^{26}$ AVA MdI-Präs 4/Dalm, Kart. 1394, MF 3, 6073-1900, David an MdI (8. 10. 1900).
} 
stellte daher beim Innenminister den Antrag zur Neusystemisierung einer Statthaltereivizepräsidentenstelle bei gleichzeitiger Einziehung eines Hofratspostens, weiters den Antrag zur Systemisierung von drei Statthaltereiratsposten gegen Einziehung einer Bezirkshauptmannstelle. Schließlich beantragte er die Systemisierung einer Statthaltereisekretärsstelle, von zwölf Bezirkskommissärstellen, von neun Statthaltereikonzipistenstellen sowie die Errichtung einer 14. Bezirkshauptmannschaft in San Pietro [Supetar] und die Reaktivierung der politischen Expositur in Crkvice. Belegbar ist die Neusystemisierung der BH San Pietro auf der Insel Brazza [Brač] im Jahre 1903.27

Zum anderen bemühte sich die Statthalterei, Studenten aufzunehmen, die im Studium bereits fortgeschritten waren. Aufnahmebedingung war, die fehlenden Prüfungen innerhalb einer Frist positiv zu absolvieren. Dies war der Fall beispielsweise bei Leonidas Franić, absolvierter Hörer der technischen Hochschule in Wien, der ausnahmsweise als Baupraktikant in den Staatsbaudienst für Dalmatien aufgenommen wurde, sich allerdings zu verpflichten hatte, innerhalb von drei Monaten die zweite Staatsprüfung aus dem Ingenieur- und Hochbaufach „mit gutem Erfolge“ abzulegen, andernfalls werde er aus dem Dienst entlassen. Enthebungen aufgrund nicht nachgebrachter Erfordernisse wurden auch in Dalmatien exekutiert.

Weiters verlieh das Ministerium seit 1894 Staatsstipendien, und zwar ausschließlich an dalmatinische Studenten des Rechts, die beide Landessprachen beherrschten und sich verpflichteten, nach Studienabschluss in den politischen Verwaltungsdienst einzutreten. ${ }^{28}$

${ }^{27}$ Ebd., Kart. 1395, MF 4, 2522-1900, David an MdI (24. 4. 1900); Kart. 1394, MF 5, 6512-1903, Statth. an MdI (11. 9. 1903); 7180-1903.

${ }^{28}$ Ebd., Kart. 1395, MF 4, 2520-1900, David an MdI (25. 4. 1900).
Die Bemühung, Beamte aus anderen Kronländern nach Dalmatien abzuwerben, war nicht erfolgreich. Hier gab es die Schwierigkeit, dass die Voraussetzung, beide Landessprachen zu beherrschen, fehlte oder es bestand bei Interessenten die Sorge, "die rechte Zeit zum Rückweg aus Dalmatien zu versäumen“. ${ }^{29}$

Der Bitte des Statthalters, von Abberufungen politischer Konzeptsbeamter aus Dalmatien abzusehen, kam das Innenministerium bedingt nach. Es sagte $z u$, diesem Wunsch im eigenen Wirkungskreis „nach Thunlichkeit Rechnung“ zu tragen und diesen auch anderen Zentralstellen mitzuteilen. ${ }^{30}$

Die Auswirkung solcher Maßnahmen bedarf noch einer genaueren Untersuchung. Zusammenfassend lässt sich aber hier bereits festhalten, dass das Innenministerium und die Statthalterei alles Mögliche taten, um das Defizit an politischem Verwaltungspersonal - auch mit Kroaten und Serben - aufzufüllen. Eine staatlich gelenkte Germanisierungstendenz ist für die Zeit ab 1900 jedenfalls nicht zu erkennen.

\section{Schlussfolgerung}

Welche Schlussfolgerung ist nun aus dem Dargestellten zu ziehen? Zunächst sei ein Vergleich gestattet. Als in Österreich im Frühling 2019 die Koalition zwischen ÖVP und FPÖ in Brüche ging, lobte Bundespräsidenten Alexander van der Bellen unmittelbar danach die „Eleganz, ja die Schönheit" der österreichischen Verfassung und merkte weiters an, es gebe keinen Grund zur Besorgnis, weil in ihr genau „verankert“ sei,

${ }^{29}$ Ebd., Kart. 1394, MF 4, 2522-1900, Statth. an MdI (24. 4. 1900).

${ }^{30}$ Ebd., MF 3, 6073-1900, Statth. an MdI (8. 10. 1900); MdI an Statth. (27. 12. 1900); MdI an HM, Ackerbaumin., MfLV, Eisenbahnmin. (27. 12. 1900); ebd., MF 4, 2715-1902. 
welche Schritte bei Regierungskrisen vorzunehmen seien. ${ }^{31}$

Lässt sich dieses Bild von der Eleganz einer Verfassung nun auch auf die österreichische staatlich-politische Verwaltung für die Zeit ab 1900 übertragen? Die lauten Stimmen der zeitgenössischen politischen Opposition hätten dies entschieden verneint (s. stellvertretend den Germanisierungsvorwurf). Auch technisch gesehen besteht ein Unterschied zur Verfassung, denn die in der Verwaltung zu unternehmenden Schritte waren nicht bis in alle Details normiert, sodass man nicht für jeden Anlass leitfadenartig vorgehen hätte können. Trotzdem kann man weitgehend von einer eleganten österreichischen Verwaltung sprechen, denn obwohl oder gerade weil die staatlich-politischen Verwaltungsorgane in Dalmatien und Wien auch Gesamtstaatsinteressen $\mathrm{zu}$ wahren hatten, waren sie weitgehend objektiv. Sie operierten grundsätzlich supranational, sie wussten mit Krisen in einem Land umzugehen, sie waren um Interessensausgleich zwischen Streitparteien bemüht, sie nahmen Mediationsaufgaben wahr oder wehrten allzu große Wünsche der militärischen Verwaltung ab. Ganz wesentlich hing der Erfolg einer „eleganten“ oder guten Verwaltung im Land vom Geist der Statthalterei ab, die sich als Scharnier zwischen den Interessen von Wien und Dalmatien verstand. Dieses österreichische Bemühen um eine gute, objektive Verwaltung, die vielen Partei- und Nationalitätenwünschen gerecht werden will, ohne das Gesamtstaatswohl aus dem Auge zu verlieren, kann nur mittels intensiven Studiums ungedruckter staatlicher Verwaltungsakten erkannt werden.

\section{Korrespondenz}

Dr. Johannes KALWODA,

Bauernfeldgasse 4/1/18

A - 1190 Wien

johannes.kalwoda@gmail.com

ORCID-Nr.: 0000-0002-4919-2547

\section{Abkürzungen}

MdI: Ministerium des Innern, Minister des Innern;

MF: Mikrofilm;

MRP: Ministerratspräsidium;

NL: Nachlass;

Präs: Präsidiale.

StPrAH: Stenographische Protokolle des Hauses der Abgeordneten des österreichischen Reichsrats.

Siehe auch das allgemeine Abkürzungsverzeichnis: [http://www.rechtsgeschichte.at/media/abk.pdf]

\section{Archive}

ÖStA, AVA, MdI-Präsidiale.

ÖStA, AVA MRP.

Státní oblastní archiv Litoměřice,

Zweigstelle Děčín, FA Thun-Hohenstein.

\footnotetext{
31 [https://www.bundespraesident.at/aktuelles/detail/ news/gespraech-mit-bundeskanzler-sebastian-kurz/] (21. 5. 2019 / 1. 10. 2019)
} 


\section{Literatur:}

Lujo BАкотіć, Srbi u Dalmaciji od pada Mletačke republike do ujedinjenja [Die Serben in Dalmatien vom Untergang der Venezianischen Republik bis zur Gründung des Jugoslawischen Staates] (Beograd 1938).

Alexander BUCZYNSKI, Der Dalmatinische Landtag; in: Helmut RuMPLER, Peter URBANITSCH (Hgg.), Die Habsburgermonarchie 1848-1918 VII/2: Verfassung und Parlamentarismus. Verfassungsrecht, Verfassungswirklichkeit. Die regionalen Repräsentativkörperschaften (Wien 2000) 1951-1981.

G. FREYTAG's erste Reichsrats-Wahlkarte 1907. Auf Grundlage des gleichen und allgemeinen Wahlrechtes ... (Wien 1907).

Hof- und Staats-Handbuch der ÖsterreichischUngarischen Monarchie für das Jahr 1912 (Wien 1912).

Aleksandar JAKIR, Dalmatien zwischen den Weltkriegen. Agrarische und urbane Lebenswelt und das Scheitern der jugoslawischen Integration (München 1999).

Ders., Marko Trogrlić, Dalmatien; in: Oliver JenS SchmitT, Michael Metzeltin (Hgg.), Das Südosteuropa der Regionen (Wien 2015) 91-132.

Johannes KALWODA, Eine engherzige Beurteilung und Würdigung der militärischen Interessen sollte man der Regierung nicht vorwerfen. Reibungspunkte zwischen militärischer und ziviler Verwaltung in Dalmatien während des Ersten Weltkriegs, in: Heeresgeschichtlichen Museum / Militärhistorischen Institut (Hg.), Beiträge zur österreichischen Militärgeschichte 864-2019 (Wien 2019) 415442.

DERS., Nationale Schlagseiten in der österreichischdalmatinischen Verwaltung im Ersten Weltkrieg? Deutungsansatz am Beispiel von Statthalterei und Bezirkshauptmännern, in: Martin KLEČACKÝ (Hg.), Úredník sluhou mnoha pánů? Nacionalizace a politizace veřejné správy ve střední Evropě 1848 1948 [Der Beamte als Diener vieler Herren? Die Nationalisierung und Politisierung der öffentli- chen Verwaltung in Mitteleuropa 1848-1948] (Praha 2018) 147-172.

DERS., Staatliche Einflussnahme bei Reichsratswahlen in Dalmatien vor wie nach der Wahlrechtsreform von 1907 und das politische Umfeld aus Statthaltereisicht, in: Thomas SIMON (Hg.), Hundert Jahre allgemeines und gleiches Wahlrecht in Österreich. Modernes Wahlrecht unter den Bedingungen eines Vielvölkerstaates (Frankfurt a.M u.a. 2010) 287-353.

DERS., Parteien, Politik und Staatsgewalt in Dalmatien (1900-1918). Zur Wechselwirkung zwischen staatlicher Verwaltung und parlamentarischer Vertretung (phil. Diss., Univ. Wien 2017).

Haira LACMANOvić-HEYDENREUTER, Dalmatien in Wien. Die dalmatinischen Abgeordneten im Wiener Reichsrat 1867-1918 (Hamburg 2011).

Ivan PEDERIN, Austrijska vlast u dalmatinskoj politici (1878.-1914.) [Österreichische Herrschaft in der dalmatinischen Politik (1878-1914)] (Zadar 2009).

Ivo PERIĆ, Dalmatinski sabor [Dalmatinischer Landtag]. 1861-1912/18 (Zadar 1978).

Günter ScHöDL, Kroatische Nationalpolitik und ,Jugoslavenstvo'. Studien zu nationaler Integration und regionaler Politik in Kroatien - Dalmatien am Beginn des 20. Jahrhunderts (München 1990).

Hermann von SCHULLERN-SCHRATTENHOFEN, Das Kolonat (Colonat) in Görz und Gradisca, in Istrien, in Dalmatien und in Tirol (Wien ${ }^{2} 1909$ ).

Eugen SCHWIEDLAND, Interministerielle Dalmatinische Kommission. Zur Kolonatsfrage. Äußerungen und Anträge des Kommissionsmitgliedes (Wien 1911).

Rok STERGAR, Nationswerdungsprozesse und neue Grenzen. Der Zusammenbruch der französischen Herrschaft in den Illyrischen Provinzen und ihre (Re-)Integration in das Kaisertum Österreich, in: Brigitte MAZOHL, Karin SchneIDER, Eva Maria WERNER (Hgg.), Am Rande der großen Politik. Italien und der Alpenraum beim Wiener Kongress (Innsbruck 2017) 97-122.

Marko Trogrlić (Hg.), Erazmo Handel, Sjećanja [Erasmus Handel, Erinnerungen] (Zagreb 2007). 